\title{
ON A CRITERION OF LOCAL INVERTIBILITY AND CONFORMALITY FOR SLICE-REGULAR QUATERNIONIC FUNCTIONS
}

ANNA GORI AND FABIO VLACCI

\begin{abstract}
A new criterion for local invertibility of slice-regular quaternionic functions is obtained. This paper is motivated by the need of finding a geometrical interpretation for analytic conditions on the real Jacobian associated to a slice-regular function $f$. The criterion involves Spherical and Cullen derivatives of $f$ and gives rise to several geometric implications including an application to related conformality properties.
\end{abstract}

2010 Mathematics Subject Classification: 30G35, 30C45

\section{Preliminaries and Introduction}

We denote by $\mathbb{H}$ the algebra of quaternions. Let $\mathbb{S}$ be the sphere of imaginary quaternions, i.e. the set of quaternions $I$ such that $I^{2}=-1$. Let $\Omega \subseteq \mathbb{H}$ be a domain.

Definition 1.1. We say that $\Omega$ is

- an axially symmetric domain if, for all $x+I y \in \Omega$, with $I \in \mathbb{S}$, the whole sphere $x+\mathbb{S} y$ is contained in $\Omega$;

- a slice domain if $\Omega \cap \mathbb{R}$ is non-empty and if given any $I \in \mathbb{S}$ the complex line $\mathbb{C}_{I}=\mathbb{R}+\mathbb{R} I$ intersected with $\Omega$ is a domain in $\mathbb{C}_{I}$.

It is possible (see [4])) to introduce a notion of regularity for functions defined in any open ball $B(0, r)=\{q \in \mathbb{H}:|q|<r\}$ (and, more in general, in some axially symmetric slice domains of $\mathbb{H}$ ) which extends the one of holomorphicity in the complex case.

Definition 1.2. If $\Omega$ is an axially symmetric slice domain in $\mathbb{H}$, a real differentiable function $f: \Omega \rightarrow \mathbb{H}$ is said to be slice-regular if, for every $I \in \mathbb{S}$, its restriction $f_{I}$ to the complex line $\mathbb{C}_{I}=\mathbb{R}+\mathbb{R} I$ passing through the origin and containing 1 and $I$ is holomorphic on $\Omega \cap \mathbb{C}_{I}$.

We recall that the notion of slice regularity was first introduced in [4]; the theory of sliceregular functions has been significantly developed in the last decade by many authors (a short list of contributions can be found in the references of [2]).

Remark 1.3. It can be proved that a function $f: B(0, r) \rightarrow \mathbb{H}$ is slice-regular in $B(0, r) \subset \mathbb{H}$ if and only if there exists a converging power series $\sum_{n} q^{n} a_{n}$ in $B(0, r)$, with $a_{n} \in \mathbb{H}$ for any $n \in \mathbb{N}$, such that $f(q)=\sum_{n} q^{n} a_{n}$ with $q \in B(0, r)$.

As a direct computation on the real components of a slice-regular function, one immediately obtains (see [4])

Partially supported by Progetto MIUR di Rilevante Interesse Nazionale PRIN 2010-11 Varietà reali e complesse: geometria, topologia e analisi armonica. The research that led to the present paper was partially supported by a grant of the group GNSAGA of Istituto Nazionale di Alta Matematica "F: Severi". 
Lemma 1.4. If $f$ is a slice-regular function on an axially symmetric slice domain $\Omega \subset \mathbb{H}$, then for every $I \in \mathbb{S}$ and for any $J \in \mathbb{S}, J \perp I$, there exist two holomorphic functions $F_{1}, F_{2}: \Omega \cap \mathbb{C}_{I} \rightarrow \mathbb{C}_{I}$ such that $f_{I}(z)=F_{1}(z)+F_{2}(z) J$ with $z=x+I y$.

For the sequel it will be important to recall a natural notion of product of polynomials (then extended to power series) which turns out to provide a "regular" multiplication of slice-regular functions when represented by converging regular power series.

Definition 1.5. Let $f(q)=\sum_{n=0}^{+\infty} q^{n} a_{n}$ and $g(q)=\sum_{n=0}^{+\infty} q^{n} b_{n}$ be given power series with coeffcients in $\mathbb{H}$ whose radii of convergence are greater than $r$. We define the regular product of $f$ and $g$ as the series $f * g(q)=\sum_{n=0}^{+\infty} q^{n} c_{n}$, where $c_{n}=\sum_{k=0}^{n} a_{k} b_{n-k}$ for all $n$, which is convergent in $B(0, r)$.

It is not difficult to see that $f * g$ is a slice-regular function defined in the open ball $B(0, r)$. Furthermore, the regular product is extended for slice-regular functions defined on a general axially symmetric domain $\Omega$ in the following way

1.1) $f * g(q)=\left\{\begin{array}{cl}0 & \text { if } f(q)=0 \\ f(q) g\left(f(q)^{-1} q f(q)\right) & \text { otherwise. }\end{array}\right.$

In the spirit of Gateaux, a notion of derivative is well-defined for slice-regular functions, namely (see [4])

Definition 1.6. Let $\Omega$ be an axially symmetric slice domain in $\mathbb{H}$ and let $f: \Omega \rightarrow \mathbb{H}$ be a slice-regular function. For any $I \in \mathbb{S}$ and any point $q=x+y I$ in $\Omega$ (with $x=\Re e q$ and $y=\Im m q)$ we define the Cullen derivative of $f$ at $q$ as

$$
\partial_{C} f(x+y I)=f^{\prime}(x+y I):=\frac{1}{2}\left(\frac{\partial}{\partial x}-I \frac{\partial}{\partial y}\right) f_{I}(x+y I)
$$

Since in $\mathbb{H}$ one can choose different imaginary units, it is also worth considering the following

Definition 1.7. Let $\Omega$ be an axially symmetric slice domain in $\mathbb{H}$ and let $f: \Omega \rightarrow \mathbb{H}$ be a slice-regular function. We define the spherical derivative of $f$ at $q$ as

$$
\partial_{S} f(q):=(q-\bar{q})^{-1}[f(q)-f(\bar{q})] .
$$

It is well known that the possibility of locally inverting a holomorphic function heavily depends on the non vanishing of the derivative; it is also clear that a holomorphic function which is locally invertible turns out to be conformal. The aim of the present paper is to investigate a generalization of these facts for quaternionic slice-regular functions.

\section{A local invertibility CRITERION}

Let $\Omega$ be an axially symmetric slice domain in $\mathbb{H}$ and $f: \Omega \rightarrow \mathbb{H}$ be a slice-regular function. If $q_{0} \in \Omega$ and $q_{0} \notin \mathbb{R}$, take $I \in \mathbb{S}$ so that $q_{o} \in \mathbb{C}_{I}$ and let $J \in \mathbb{S}$ such that $I \perp J$ as vectors in $\mathbb{R}^{3}$. According to this choice of local coordinates, consider the corresponding splittings

$$
f_{I}=F_{1}+F_{2} J \quad \text { and } \quad R_{q_{0}} f=R_{1}+R_{2} J
$$

where $R_{q_{0}} f$ is defined by 


$$
f(q)-f\left(q_{o}\right)=\left(q-q_{0}\right) * R_{q_{0}} f(q) .
$$

1 We also recall here that

$$
R_{q_{0}} f\left(q_{0}\right)=\partial_{C} f\left(q_{0}\right) \quad \text { and } \quad R_{q_{0}} f\left(\overline{q_{0}}\right)=\partial_{S} f\left(q_{0}\right) .
$$

2 Furthermore, from Theorem 8.16 in [2] and using the local coordinates as a above, the

3 (complex) Jacobian of $f$ at $q_{0}$ can be written as

$$
d f_{q_{0}}=\left(\begin{array}{ll}
R_{1}\left(q_{0}\right) & -\overline{R_{2}\left(\overline{q_{0}}\right)} \\
R_{2}\left(q_{0}\right) & \overline{R_{1}\left(\overline{q_{0}}\right)}
\end{array}\right)
$$

4 We observe first that if $f$ is a slice-preserving function (i.e. if $f$ maps $\mathbb{C}_{I} \cap \Omega$ into $\mathbb{C}_{I}$ ) then,

5 in local coordinates, $f=F_{1}$ and $R_{f}=R_{1}$, and hence

$$
d f_{q_{0}}=\left(\begin{array}{cc}
R_{1}\left(q_{o}\right) & 0 \\
0 & R_{1}\left(q_{o}\right)
\end{array}\right)
$$

6 which means that the complex Jacobian is invertible if and only if $R_{1}\left(q_{o}\right) \neq 0$ or, equivalently, 7 if and only if $\partial_{C} f\left(q_{o}\right) \neq 0$. In general, for a $2 \times 2$ quaternionic matrix, its invertibility depends 8 on the non vanishing of its Dieudonné determinant det $_{\mathbb{H}}$ which is defined as follows

$$
\operatorname{det}_{\mathbb{H}}\left(\begin{array}{ll}
a & b \\
c & d
\end{array}\right)=\left\{\begin{array}{lll}
-b c & \text { if } & a=0 \\
a d-a b a^{-1} c & \text { if } & a \neq 0
\end{array}\right.
$$

9 In the case of the Jacobian of $f$ at $q_{0}$, we observe that $R_{1}, R_{2}, \overline{R_{1}}$ and $\overline{R_{2}}$ are all self-maps 10 of $\Omega \cap \mathbb{C}_{I}$ and hence their products commute; in other words

$$
\left.\operatorname{det}_{\mathbb{H}}\left(\begin{array}{ll}
R_{1}\left(q_{0}\right) & -\overline{R_{2}\left(\overline{q_{0}}\right)} \\
R_{2}\left(q_{0}\right) & \overline{R_{1}\left(\overline{q_{0}}\right)}
\end{array}\right)=R_{1} q_{0}\right) \overline{R_{1}\left(\overline{q_{0}}\right)}+R_{2}\left(q_{0}\right) \overline{R_{2}\left(\overline{q_{0}}\right)} .
$$

11 Therefore, according to the previous positions, one can write

$$
\partial_{c} f\left(q_{0}\right)=\left(\begin{array}{l}
R_{1}\left(q_{0}\right) \\
R_{2}\left(q_{0}\right)
\end{array}\right) \quad \partial_{S} f\left(q_{0}\right)=\left(\begin{array}{l}
R_{1}\left(\overline{q_{0}}\right) \\
R_{2}\left(\overline{q_{0}}\right)
\end{array}\right)
$$

12 and hence, using the (standard) Hermitian product $\langle\cdot \mid \cdot\rangle$ in $\mathbb{C}^{2}$, one obtains that

$$
\operatorname{det}_{\mathbb{H}}\left(\begin{array}{ll}
R_{1}\left(q_{0}\right) & -\overline{R_{2}\left(\overline{q_{0}}\right)} \\
R_{2}\left(q_{0}\right) & \overline{R_{1}\left(\overline{q_{0}}\right)}
\end{array}\right)=\left\langle\partial_{C} f\left(q_{0}\right) \mid \partial_{S} f\left(q_{0}\right)\right\rangle .
$$

13 Remark 2.1. The usual quaternionic Hermitian product $\langle\cdot, \cdot\rangle_{\mathbb{H}}$ is defined as

$$
\langle q, w\rangle_{\mathbb{H}}=q \cdot \bar{w} .
$$

14 If one considers $q=a+b J$ and $w=c+d J$, with $a, b, c, d \in \mathbb{C}_{I}$ and $J \perp I$, then an 15 easy and direct computation shows that $\langle q, w\rangle_{\mathbb{H}}$ splits as the sum of a component along $\mathbb{C}_{I}$ 16 (namely $a \bar{c}+b \bar{d})$ and another component in $\mathbb{C}_{I}^{\perp}$. The component along $\mathbb{C}_{I}$ coincides with the 17 Hermitian product $\langle\cdot \mid \cdot\rangle$ defined above.

18 Remark 2.2. We recall that in [5] the same Hermitian product of the Cullen and Spherical derivatives of a slice-regular function $f$ appears in conditions which guarantee starlikeness 20 for the function $f$.

21 We summarize our considerations by stating the following criterion of local invertibility 
1 Proposition 2.3. With the above-given notation,

$$
d f_{q_{0}} \text { is locally invertible } \Longleftrightarrow\left\langle\partial_{C} f\left(q_{0}\right) \mid \partial_{S} f\left(q_{0}\right)\right\rangle \neq 0 .
$$

2 Remark 2.4. The previous proposition can be interpreted in terms of Remark 2.1. Thus if $3 q_{0} \in \mathbb{C}_{I}$, with the above notation, $d f_{q_{0}}$ is not invertible if and only if the $\left\langle\partial_{C} f\left(q_{0}\right) \mid \partial_{S} f\left(q_{0}\right)\right\rangle_{\mathbb{H}}$

4 belongs to $\mathbb{C}_{I}^{\perp}$ in accordance with the results in [1] which generalize the ones in [3].

5 An immediate consequence of the previous proposition is the obvious result that $f$ is 6 not locally invertible if $\partial_{C} f$ or $\partial_{S} f$ vanish. The fact that $\partial_{C} f\left(q_{0}\right)=0$ implies non-local 7 invertibility for $f$ is well-known and clear as in the holomorphic case.

8 On the other hand, if $q_{0}=x_{0}+I y_{0}$ and given any $q=x_{0}+J y_{0}$ with $J \in \mathbb{S}$ (see [2]), it 9 turns out that

$$
f(q)=c+J b
$$

10 with the same $b, c$ for any $q \in \mathbb{S}_{q_{0}}:=\left\{x_{0}+J y_{0}: J \in \mathbb{S}\right\}$ and $b=\partial_{s} f\left(q_{0}\right)$; then it 11 clearly follows that if $\partial_{S} f\left(q_{0}\right)=0$, the function $f$ is constant on the sphere $\mathbb{S}_{q_{0}}$, and so $f$ 12 is not invertible. In order to provide an example of a slice-regular function whose Cullen 13 and spherical derivatives don't vanish at $q_{0}$ but their Hermitian product does, we recall the 14 following fact (see [2]): the Jacobian of $f$ is not invertible at $q_{0}=x_{0}+I y_{0}$ if and only if 15 there exist $\widetilde{q_{0}}=x_{0}+I_{1} y_{0}$ and a slice-regular function $g$ such that

$$
f(q)-f\left(q_{o}\right)=\left(q-q_{0}\right) *\left(q-\widetilde{q_{0}}\right) * g(q) .
$$

and

$$
R_{q_{0}} f(q)=\left(q-\widetilde{q_{0}}\right) * g(q) .
$$

Assume now that (with the usual frame associated to the choice of $J \perp I$ ) we choose the restriction of the slice-regular function $g$ along the slice $\mathbb{C}_{I}$ to be $g_{I}(q)=q+q^{2} J$ and take $\widetilde{q}_{0}=x_{0}+J y_{0}$; thus, in this case, the restriction of the slice-regular function $R_{q_{0}} f(q)$ along the slice $\mathbb{C}_{I}$ is $\left(q-\widetilde{q_{0}}\right) *\left(q+q^{2} J\right)$ and, in particular, $R_{1}(q)=q^{2}-q x_{0}+q^{2} y_{0}$ and $R_{2}(q)=q^{3}-q y_{0}-q^{2} x_{0}$. Hence

$$
R_{1}\left(q_{0}\right)=q_{0}^{2}-q_{0} x_{0}+q_{0}^{2} y_{0}=-y_{0}^{2}-y_{0}^{3}+x_{0}^{2} y_{0}+\left(x_{0} y_{0}+2 x_{0} y_{0}^{2}\right) I
$$

$$
R_{2}\left(q_{0}\right)=q_{0}^{3}-q_{0} y_{0}-q_{0}^{2} x_{0}=-x_{0} y_{0}-2 x_{0} y_{0}^{2}+\left(-y_{0}^{2}-y_{0}^{3}+x_{0}^{2} y_{0}\right) I
$$

or

$$
R_{2}\left(q_{0}\right)=-I R_{1}\left(q_{0}\right) .
$$

Furthermore, as easily seen from direct computations,

$$
R_{1}\left(\overline{q_{0}}\right)=-y_{0}^{2}-y_{0}^{3}+x_{0}^{2} y_{0}-\left(x_{0} y_{0}+2 x_{0} y_{0}^{2}\right) I=\overline{R_{1}\left(q_{0}\right)}
$$

and

$$
R_{2}\left(\overline{q_{0}}\right)=-x_{0} y_{0}-2 x_{0} y_{0}^{2}+\left(y_{0}^{2}+y_{0}^{3}-x_{0}^{2} y_{0}\right) I=\overline{R_{2}\left(q_{0}\right)}
$$

$$
\begin{aligned}
& \operatorname{det}_{\mathbb{H}}\left(\begin{array}{ll}
R_{1}\left(q_{0}\right) & -\overline{R_{2}\left(\overline{q_{0}}\right)} \\
R_{2}\left(q_{0}\right) & \overline{R_{1}\left(\overline{q_{0}}\right)}
\end{array}\right)=R_{1}\left(q_{0}\right) \overline{R_{1}\left(\overline{q_{0}}\right)}+R_{2}\left(q_{0}\right) \overline{R_{2}\left(\overline{q_{0}}\right)}= \\
& =\left\langle\partial_{c} f\left(q_{0}\right) \mid \partial_{S} f\left(q_{0}\right)\right\rangle=R_{1}\left(q_{0}\right)^{2}+R_{2}\left(q_{0}\right)^{2}=R_{1}\left(q_{0}\right)^{2}-R_{1}\left(q_{0}\right)^{2}=0
\end{aligned}
$$


5 for all $u \in \mathbb{C}_{I}$ and $v \in \mathbb{C}_{I}^{\perp}$,

$$
d f_{q_{o}}(u+w)=u \partial_{C} f\left(q_{0}\right)+w \partial_{S} f\left(q_{0}\right) .
$$

$6 \quad$ We'll assume $w=v J$ with $J \perp I$. Since, using the frame associated to the splitting 7 corresponding to the choice of $J \perp I$, one has

$$
\begin{aligned}
& \partial_{C} f\left(q_{0}\right)=R_{1}\left(q_{0}\right)+R_{2}\left(q_{0}\right) J \\
& \partial_{S} f\left(q_{0}\right)=R_{1}\left(\overline{q_{0}}\right)+R_{2}\left(\overline{q_{0}}\right) J,
\end{aligned}
$$

8 then

$$
\begin{aligned}
d f_{q_{o}}(u+v J) & =u R_{1}\left(q_{0}\right)+v J R_{2}\left(\overline{q_{o}}\right) J+u R_{2}\left(q_{0}\right) J+v J R_{1}\left(\overline{q_{o}}\right) \\
& =u R_{1}\left(q_{0}\right)-v \overline{R_{2}\left(\overline{q_{o}}\right)}+\left[u R_{2}\left(q_{0}\right)+v \overline{R_{1}\left(\overline{q_{o}}\right)}\right] J .
\end{aligned}
$$

9 Therefore, after some computations,

10

$$
\begin{aligned}
\left|d f_{q_{o}}(u+v J)\right|^{2} & =\left\langle d f_{q_{o}}(u+v J) \mid d f_{q_{o}}(u+v J)\right\rangle= \\
& =|u|^{2}\left|R_{1}\left(q_{0}\right)\right|^{2}+|v|^{2}\left|\overline{R_{2}\left(\overline{q_{o}}\right)}\right|^{2}+u\left[R_{2}\left(q_{0}\right) R_{1}\left(\overline{q_{o}}\right)-R_{1}\left(q_{0}\right) R_{2}\left(\overline{q_{o}}\right)\right] \bar{v}+ \\
& +v\left[\overline{R_{1}\left(\overline{q_{0}}\right)} \overline{R_{2}\left(q_{o}\right)}-\overline{R_{2}\left(\overline{q_{0}}\right)} \overline{R_{1}\left(q_{o}\right)}\right] \bar{u}+|u|^{2}\left|R_{2}\left(q_{0}\right)\right|^{2}+|v|^{2}\left|\overline{R_{1}\left(\overline{q_{o}}\right)}\right|^{2}= \\
& =|u|^{2}\left|\partial_{C} f\left(q_{0}\right)\right|^{2}+|v|^{2}\left|\partial_{S} f\left(q_{0}\right)\right|^{2}+ \\
& +u\left[R_{2}\left(q_{0}\right) R_{1}\left(\overline{q_{o}}\right)-R_{1}\left(q_{0}\right) R_{2}\left(\overline{q_{o}}\right)\right] \bar{v}+v\left[\overline{R_{1}\left(\overline{q_{0}}\right)} \overline{R_{2}\left(q_{o}\right)}-\overline{R_{2}\left(\overline{q_{0}}\right)} \overline{R_{1}\left(q_{o}\right)}\right] \bar{u} .
\end{aligned}
$$

In other words, if $\mathcal{A}=u\left[R_{2}\left(q_{0}\right) R_{1}\left(\overline{q_{o}}\right)-R_{1}\left(q_{0}\right) R_{2}\left(\overline{q_{o}}\right)\right] \bar{v}$, one gets

$$
\begin{aligned}
\left|d f_{q_{o}}(u+v J)\right|^{2} & =|u|^{2}\left|\partial_{C} f\left(q_{0}\right)\right|^{2}+|v|^{2}\left|\partial_{S} f\left(q_{0}\right)\right|^{2}+\mathcal{A}+\overline{\mathcal{A}}= \\
& =|u|^{2}\left|\partial_{C} f\left(q_{0}\right)\right|^{2}+|v|^{2}\left|\partial_{S} f\left(q_{0}\right)\right|^{2}+2 \Re e \mathcal{A} ;
\end{aligned}
$$

therefore if $\partial_{C} f\left(q_{0}\right) \neq 0$ and $\partial_{S} f\left(q_{0}\right) \neq 0$, there exists no pair $(u, v) \neq(0,0)$ such that $d f_{q_{0}}(u+v J)=0$ if $\Re e(\mathcal{A}) \geq 0$. In this case, $f$ is then locally invertible. On the other hand, if $\partial_{C} f\left(q_{0}\right)=\partial_{S} f\left(q_{0}\right)=0$, then $\mathcal{A}=0$. In this case, $f$ is not locally invertible.

Now we want to investigate what happens to $\Re e \mathcal{A}$ when $\left\langle\partial_{C} f\left(q_{0}\right) \mid \partial_{S} f\left(q_{0}\right)\right\rangle=0$ but $\partial_{C} f\left(q_{0}\right) \neq 0$ and $\partial_{S} f\left(q_{0}\right) \neq 0$. First of all, one can write

$$
\mathcal{A}=u \mathcal{B} \bar{v}
$$

18 where

$$
\mathcal{B}:=R_{2}\left(q_{0}\right) R_{1}\left(\overline{q_{o}}\right)-R_{1}\left(q_{0}\right) R_{2}\left(\overline{q_{o}}\right) .
$$

We observe that 


$$
\operatorname{det}_{\mathbb{H}}\left(\begin{array}{ll}
R_{1}\left(\overline{q_{0}}\right) & R_{1}\left(q_{0}\right) \\
R_{2}\left(\overline{q_{0}}\right) & R_{2}\left(q_{0}\right)
\end{array}\right)=\mathcal{B} .
$$

1 It then turns out that $\mathcal{B}=0$ if and only if $\partial_{C} f\left(q_{0}\right)$ and $\partial_{S} f\left(q_{0}\right)$ are linearly dependent. If 2 one assumes that $\partial_{C} f\left(q_{0}\right) \neq 0$ and $\partial_{S} f\left(q_{0}\right) \neq 0$ and $\left\langle\partial_{C} f\left(q_{0}\right) \mid \partial_{S} f\left(q_{0}\right)\right\rangle=0$, then $\partial_{C} f\left(q_{0}\right)$ 3 and $\partial_{S} f\left(q_{0}\right)$ are linearly independent, so that $\mathcal{B} \neq 0$. Furthermore, we state the following

4 Proposition 3.1. Let $f$ be a slice-regular function on an axialy symmetric slice domain $\Omega$ 5 and let $q_{o}$ be in $\Omega$, If $\partial_{C} f\left(q_{0}\right) \neq 0$ and $\partial_{S} f\left(q_{0}\right) \neq 0$, then (with the notations introduced so far) the following conditions are equivalent

- $f$ is not locally invertible in (a neighborhood of) $q_{0}$;

- the matrix associated to $d f_{q_{0}}$ is not invertible or singular;

- $\left\langle\partial_{C} f\left(q_{0}\right) \mid \partial_{S} f\left(q_{0}\right)\right\rangle=0$

- the Hermitian matrix

$$
\left(\begin{array}{cc}
\left|\partial_{c} f\left(q_{0}\right)\right|^{2} & \overline{\mathcal{B}} \\
\mathcal{B} & \left|\partial_{S} f\left(q_{0}\right)\right|^{2}
\end{array}\right)
$$

is singular or the associated Hemitian product is degenerate.

Proof. Thee first two conditions are clearly equivalent and they both are equivalent to the third condition, thanks to Proposition 2.3. Thus, under the assumptions $\partial_{C} f\left(q_{0}\right) \neq 0$ and $\partial_{S} f\left(q_{0}\right) \neq 0$, the condition $0=\left\langle\partial_{C} f\left(q_{0}\right) \mid \partial_{S} f\left(q_{0}\right)\right\rangle=R_{1}\left(q_{0}\right) \overline{R_{1}\left(\overline{q_{0}}\right)}+R_{2}\left(q_{0}\right) \overline{R_{2}\left(\overline{q_{0}}\right)}$ implies that at least one of the following identities holds

$$
R_{1}\left(q_{0}\right)=\frac{-R_{2}\left(q_{0}\right) \overline{R_{2}\left(\overline{q_{0}}\right)}}{\overline{R_{1}\left(\overline{q_{0}}\right)}} \quad R_{2}\left(q_{0}\right)=\frac{-R_{1}\left(q_{0}\right) \overline{R_{1}\left(\overline{q_{0}}\right)}}{\overline{R_{2}\left(\overline{q_{0}}\right)}} .
$$

Let us assume that the first one holds, so that, after substitution,

$$
\mathcal{B}=\frac{R_{2}\left(q_{0}\right)}{\overline{R_{1}\left(\overline{q_{0}}\right)}}\left|\partial_{S} f\left(q_{0}\right)\right|^{2}
$$

and hence

$$
\left|d f_{q_{0}}(u+v J)\right|^{2}=0 \Longleftrightarrow u \frac{R_{2}\left(q_{0}\right)}{\overline{R_{1}\left(\overline{q_{0}}\right)}} \bar{v}+v \frac{\overline{R_{2}\left(q_{0}\right)}}{R_{1}\left(\overline{q_{0}}\right)} \bar{u}+|u|^{2} \frac{\left|\partial_{C} f\left(q_{0}\right)\right|^{2}}{\left|\partial_{S} f\left(q_{0}\right)\right|^{2}}+|v|^{2}=0 .
$$

18 This equation can be regarded as the equation which describes ker $d f_{q_{0}}$. Another way to 19

$$
(u, v)\left(\begin{array}{cc}
\left|\partial_{c} f\left(q_{0}\right)\right|^{2} & \overline{\mathcal{B}} \\
\mathcal{B} & \left|\partial_{S} f\left(q_{0}\right)\right|^{2}
\end{array}\right)\left(\begin{array}{l}
\bar{u} \\
\bar{v}
\end{array}\right)=0
$$

We observe that the matrix in (3.1) is Hermitian, so that it defines a Hermitian product. Therefore, there exists a pair $(u, v) \neq(0,0)$ such that $d f_{q_{0}}(u+v J)=0$ (or, equivalently, $d f_{q_{0}}$ is not invertible) if and only if the Hermitian product introduced in (3.1) is degenerate. Indeed, this is equivalent to saying that

$$
\operatorname{det}_{\mathbb{H}}\left(\begin{array}{cc}
\left|\partial_{c} f\left(q_{0}\right)\right|^{2} & \overline{\mathcal{B}} \\
\mathcal{B} & \left|\partial_{S} f\left(q_{0}\right)\right|^{2}
\end{array}\right)=\left|\partial_{c} f\left(q_{0}\right)\right|^{2}\left|\partial_{S} f\left(q_{0}\right)\right|^{2}-|\mathcal{B}|^{2}=0 .
$$


1 Now, in general, one has

$$
\begin{aligned}
|\mathcal{B}|^{2} & =\left[R_{2}\left(q_{0}\right) R_{1}\left(\overline{q_{o}}\right)-R_{1}\left(q_{0}\right) R_{2}\left(\overline{q_{o}}\right)\right]\left[\overline{R_{1}\left(\overline{q_{o}}\right)} \overline{R_{2}\left(q_{0}\right)}-\overline{R_{2}\left(\overline{q_{o}}\right)} \overline{R_{1}\left(q_{0}\right)}\right]= \\
& =\left|R_{2}\left(q_{0}\right)\right|^{2}\left|R_{1}\left(\overline{q_{o}}\right)\right|^{2}+\left|R_{1}\left(q_{0}\right)\right|^{2}\left|R_{2}\left(\overline{q_{o}}\right)\right|^{2}-R_{2}\left(q_{0}\right) R_{1}\left(\overline{q_{o}}\right) \overline{R_{2}\left(\overline{q_{o}}\right)} \overline{R_{1}\left(q_{0}\right)}+ \\
& -R_{1}\left(q_{0}\right) R_{2}\left(\overline{q_{o}}\right) \overline{R_{1}\left(\overline{q_{o}}\right)} \overline{R_{1}\left(q_{0}\right)}
\end{aligned}
$$

2 If $0=\left\langle\partial_{C} f\left(q_{0}\right) \mid \partial_{S} f\left(q_{0}\right)\right\rangle=R_{1}\left(q_{0}\right) \overline{R_{1}\left(\overline{q_{0}}\right)}+R_{2}\left(q_{0}\right) \overline{R_{2}\left(\overline{q_{0}}\right)}$ then $R_{1}\left(q_{0}\right) \overline{R_{1}\left(\overline{q_{0}}\right)}=-R_{2}\left(q_{0}\right) \overline{R_{2}\left(\overline{q_{0}}\right)}$ 3 and since $R_{1}, \overline{R_{1}}, R_{2}$ and $\overline{R_{2}}$ commute, one can equivalently write

$$
|\mathcal{B}|^{2}=\left|R_{2}\left(q_{0}\right)\right|^{2}\left|R_{1}\left(\overline{q_{o}}\right)\right|^{2}+\left|R_{1}\left(q_{0}\right)\right|^{2}\left|R_{2}\left(\overline{q_{o}}\right)\right|^{2}+\left|R_{1}\left(q_{0}\right)\right|^{2}\left|R_{1}\left(\overline{q_{o}}\right)\right|^{2}+\left|R_{2}\left(q_{0}\right)\right|^{2}\left|R_{2}\left(\overline{q_{o}}\right)\right|^{2}
$$

4 so that

5 which implies that

$$
|\mathcal{B}|^{2}=\left|\partial_{c} f\left(q_{0}\right)\right|^{2}\left|\partial_{S} f\left(q_{0}\right)\right|^{2}
$$

$$
\left|d f_{q_{0}}(u+v J)\right|^{2}=0
$$

6 has a solution $(u, v) \neq(0,0)$ or $d f_{q_{0}}$ is singular, as desired.

$7 \quad$ We conclude this paper by providing an explicit description of ker $d f_{q_{0}}$ under the assump8 tions $\partial_{C} f\left(q_{0}\right) \neq 0, \partial_{S} f\left(q_{0}\right) \neq 0$ and $\left\langle\partial_{C} f\left(q_{0}\right) \mid \partial_{S} f\left(q_{0}\right)\right\rangle=0$. It is known (see [2]) that in 9 general the rank of $d f_{q_{0}}$ (regarded as a $4 \times 4$ real matrix) can be 0,2 or 4 . We'll show in 10 detail that under the assumptions $\partial_{C} f\left(q_{0}\right) \neq 0, \partial_{S} f\left(q_{0}\right) \neq 0$ and $\left\langle\partial_{C} f\left(q_{0}\right) \mid \partial_{S} f\left(q_{0}\right)\right\rangle=0$, the 11 rank of $d f_{q_{0}}$ is precisely 2 .

12 First of all, we'll write $u=t+s I, v=x+y I$ and

$$
\mathcal{B}=B_{1}+B_{2} I \quad \overline{\mathcal{B}}=B_{1}-B_{2} I .
$$

13 We recall that, under our assumptions,

$$
|\mathcal{B}|^{2}=B_{1}^{2}+B_{2}^{2}=\left|\partial_{c} f\left(q_{0}\right)\right|^{2}\left|\partial_{S} f\left(q_{0}\right)\right|^{2} .
$$

14 Hence the equation of ker $d f_{q_{0}}$ as in (3.1) becomes

$$
(s+t I, x+y I)\left(\begin{array}{cc}
\left|\partial_{c} f\left(q_{0}\right)\right|^{2} & B_{1}-I B_{2} \\
B_{1}+B_{2} I & \left|\partial_{S} f\left(q_{0}\right)\right|^{2}
\end{array}\right)\left(\begin{array}{c}
s-t I \\
x-y I
\end{array}\right)=0 ;
$$

15 after some computations, one obtains

$$
\left(t^{2}+s^{2}\right)\left|\partial_{c} f\left(q_{0}\right)\right|^{2}+\left(x^{2}+y^{2}\right)\left|\partial_{S} f\left(q_{0}\right)\right|^{2}+2 x B_{1} t-2 y B_{2} t+2 y B_{1} s+2 x B_{2} s=0
$$

16 or

$$
\left(t^{2}+s^{2}\right) \frac{\left(B_{1}^{2}+B_{2}^{2}\right)}{\left|\partial_{S} f\left(q_{0}\right)\right|^{4}}+x^{2}+y^{2}+2 x \frac{\left(B_{1} t+B_{2} s\right)}{\left|\partial_{S} f\left(q_{0}\right)\right|^{2}}+2 y \frac{\left(B_{1} s-B_{2} t\right)}{\left|\partial_{S} f\left(q_{0}\right)\right|^{2}}=0 .
$$

17 This leads to write

18 or

$$
\left[x+\frac{\left(B_{1} t+B_{2} s\right)}{\left|\partial_{S} f\left(q_{0}\right)\right|^{2}}\right]^{2}+\left[y+\frac{\left(B_{1} s-B_{2} t\right)}{\left|\partial_{S} f\left(q_{0}\right)\right|^{2}}\right]^{2}=0
$$

$$
x=-\frac{\left(B_{1} t+B_{2} s\right)}{\left|\partial_{S} f\left(q_{0}\right)\right|^{2}} \quad y=-\frac{\left(B_{1} s-B_{2} t\right)}{\left|\partial_{S} f\left(q_{0}\right)\right|^{2}}
$$


1 Therefore, the set of pairs $(u, v)$ such that $d f_{q_{0}}(u+v J)=0$ is a plane in $\mathbb{R}^{4}$, and so the rank 2 of the real $4 \times 4$ matrix associated to $d f_{q_{0}}$ is 2 , as expected.

3 Remark 3.2. From the above-given calculations it also follows that a slice-regular quater4 nionic function turns out to be conformal at $q_{0}$ (in the real sense, as a function from $\Omega \subseteq \mathbb{R}^{4}$ $\left.5 \rightarrow \mathbb{R}^{4}\right)$ if and only if $\mathcal{B}=0$ and $\left|\partial_{C} f\left(q_{0}\right)\right|^{2}=\left|\partial_{S} f\left(q_{0}\right)\right|^{2} \neq 0$. This is for instance the case of 6 a slice-regular function $f$ whose associated (slice-regular) function $R_{q_{0}} f$ is real analytic (i.e. $7 \quad R_{q_{0}} f(q)=\sum_{n} q^{n} a_{n}$ with $a_{n} \in \mathbb{R}$ for any $\left.n \in \mathbb{N}\right)$. The real analyticity of $R_{q_{0}} f$ is clearly a 8 consequence of the real analyticity of $f$ together with the assumption $q_{0} \in \mathbb{R}$ (which implies 9 that $f$ is a slice-preserving and slice-regular quaternionic function) but one can consider 10 also other functions such as the following

$$
f(q)=J+(q-I) * \operatorname{Exp}(q)
$$

11 where $q_{0}=I \in \mathbb{S}, f(I)=J \neq I, J \in \mathbb{S}$ and $\operatorname{Exp}(q)=\sum_{n} \frac{q^{n}}{n !}$. The function $f$ turns out to be not slice-preserving but conformal at $q_{0}=I$. On the other hand, if one drops the assumptions $q_{0} \in \mathbb{R}$ it is not in general true that, for a slice-regular and slice-preserving function $f$, the (associated) slice-regular function $R_{q_{0}} f$ is real analytic, as the function

$$
f(q)=q^{2}-2 q \Re e q_{0}+\left|q_{0}\right|^{2}=\left(q-q_{0}\right) *\left(q-\overline{q_{0}}\right)
$$

clearly demonstrates.

From the previous remark and considerations, we conclude by stating this interesting property on the Cullen and Spherical derivatives of a slice-regular function which turns out to be also conformal.

Corollary 3.3. Assume that $f: \Omega \subseteq \mathbb{H} \rightarrow \mathbb{H}$ is a slice-regular function. If $f$ is confromal at $q_{0} \in \Omega$ then there exist two unitary quaternions $U, V \in \mathbb{H}$, with $|U|=|V|=1$, such that

$$
\partial_{C} f\left(q_{0}\right)=U \partial_{S} f\left(q_{0}\right) V
$$

\section{REFERENCES}

[1] A. Altavilla, On the real differential of a slice regular function, Adv. in Geom., to appear

[2] G. Gentili, C. Stoppato, D. Struppa, Regular functions of a quaternionic variable, Springer Monographs in Mathematics, Springer, Heidelberg, 2013.

[3] G. Gentili, s. Salamon, C. Stoppato, Twistor transfos of quaternionic functions and orthogonal complex structures, J. Eur. Math. Soc. (JEMS), 16(11), 2323-2353 (2014).

[4] G. Gentili, D. Struppa, A new theory of regular functions of a quaternionic variable, Adv. Math., 216, 279-301 (2007).

[5] A. Gori, F. Vlacci, Starlikeness for functions of a hypercomplex variable Proc. Amer.Math.Soc., 145 (2), 791-804 (2017).

Dipartimento di Matematica - Università di Milano, Via Saldini 50, 20133 Milano Italy

E-mail address: anna.gori@unimi.it

Dipartimento di Matematica e Informatica "U. Dini" - Università di Firenze Viale MorGAGNi 67/A, 50134 FirenZe ItALY

E-mail address: vlacci@math.unifi.it 